\title{
CONJUGATION AND EXCESS IN THE STEENROD ALGEBRA
}

\author{
JUDITH H. SILVERMAN
}

(Communicated by Thomas Goodwillie)

ABSTRACT. In this paper we prove a formula involving the canonical antiautomorphism $\chi$ of the mod-2 Steenrod algebra $\mathscr{A}(2)$, namely,

$$
\begin{aligned}
& \chi\left(S q^{2^{j}\left(2^{i+1}-1\right)} S q^{2^{j-1}\left(2^{i+1}-1\right)} \cdots S q^{\left(2^{i+1}-1\right)}\right) \\
& \quad=S q^{2^{i}\left(2^{j+1}-1\right)} S q^{2^{i-1}\left(2^{j+1}-1\right)} \ldots S q^{\left(2^{j+1}-1\right)},
\end{aligned}
$$

and discuss its implications for the study of the image of the $\mathscr{A}(2)$-action on $\mathbb{F}_{2}\left[x_{1}, \ldots, x_{s}\right]$.

\section{INTRODUCTION}

1.1. Notation. The Milnor basis of the mod-2 Steenrod algebra is indexed by sequences $R=\left(r_{1}, r_{2}, \ldots\right)$ of nonnegative integers almost all of which are $0[\mathrm{M}]$. We denote the corresponding basis element by $\langle R\rangle$; its dimension is $|\langle R\rangle|=\sum_{i}\left(2^{i}-1\right) r_{i}$. If $R=(r, 0,0, \ldots)$, then the corresponding basis element, abbreviated $\langle r\rangle$, is the Steenrod square $S q^{r}$. The element $\left\langle a_{1}\right\rangle \cdots\left\langle a_{n}\right\rangle$ is admissible if $a_{r} \geq 2 a_{r+1}$ for $r<n$ and $a_{n}>0$ if $n>1$. The admissible elements form an additive basis of $\mathscr{A}(2)$ [SE], but though the objects of study in this paper are certain admissible elements, the calculations are best expressed in terms of the Milnor basis. In particular, define $\mathscr{B}(\theta)$ for $\theta \in \mathscr{A}(2)$ to be the set of Milnor basis elements appearing in $\theta$, so that $\theta=\sum\{\langle R\rangle:\langle R\rangle \in \mathscr{B}(\theta)\}$.

The Steenrod algebra acts on $\mathbb{F}_{2}\left[x_{1}, \ldots, x_{s}\right]$, the polynomial algebra on elements $x_{i}$ of dimension 1, which is the mod-2 cohomology rixg of

$$
\overbrace{\mathbb{R} P^{\infty} \times \cdots \times \mathbb{R} P^{\infty}}^{s} .
$$

Following Singer [Si], we say that a polynomial $F$ is hit it it is the image of the positively graded part of $\mathscr{A}(2)$, that is, if $F=\sum_{i>0} S q^{i} F_{i}$ for some polynomials $F_{i}[\mathrm{Pe}, \mathrm{W}]$. The excess of an element $\theta \in \mathscr{A}(2)$ is given by $\operatorname{ex}(\theta)=\min \{\hat{s}$ : $\left.\theta\left(x_{1} x_{2} \cdots x_{\hat{s}}\right) \neq 0 \in \mathbb{F}_{2}\left[x_{1}, \ldots, x_{\hat{s}}\right]\right\}$ (cf. [K]). Since linear maps commute with the action of $\mathscr{A}(2)$, it follows that whatever $s$ might be, $\theta(F)=0$ for any polynomial in $\mathbb{F}_{2}\left[x_{1}, \ldots, x_{s}\right]$ of degree $<\operatorname{ex}(\theta)$. One has that $\operatorname{ex}(\langle n\rangle)=n$

Received by the editors February 26, 1992.

1991 Mathematics Subject Classification. Primary 55S10; Secondary 55R40.

Key words and phrases. Steenrod algebra, canonical automorphism, excess, Steenrod algebra action on polynomial algebra. 
and, more generally, that $\operatorname{ex}\left(\left\langle a_{1}\right\rangle \cdots\left\langle a_{n}\right\rangle\right)=\sum_{r=1}^{n-1}\left(a_{r}-2 a_{r+1}\right)+a_{n}$ if $\left\langle a_{1}\right\rangle \cdots\left\langle a_{n}\right\rangle$ is admissible [SE].

Given a pair $(k, a)$ of nonnegative integers, we define $T(k ; a)=\left\langle 2^{k} a\right\rangle \ldots$ $\langle 2 a\rangle\langle a\rangle$. Note that $|T(k ; a)|=\left(2^{k+1}-1\right) a$, that $T(k ; a)$ is admissible of excess $a$, and that on polynomials of degree $|A|=a$ one has $T(k ; a)(A)=A^{2^{k+1}}$.

We denote by $\chi$ the canonical antiautomorphism of $\mathscr{A}(2)$.

1.2. Results. In [D] Davis computes $\chi T(j ; 1)$ :

Theorem 1.1 [D]. For all $j \geq 0$, we have $\chi T(j ; 1)=\left\langle 2^{j+1}-1\right\rangle$.

Our Theorem 3.1 is a generalization of Theorem 1.1.

Theorem 3.1. For all integers $i$ and $j$, we have $\chi T\left(j ; 2^{i+1}-1\right)=T\left(i ; 2^{j+1}-1\right)$.

Davis's argument proves also that $\chi T(j ; 2)=\left\langle 2\left(2^{j+1}-1\right)\right\rangle$, leading one to conjecture the following.

Conjecture 1.2. $\chi T\left(j ; 2\left(2^{i+1}-1\right)\right)=T\left(i ; 2\left(2^{j+1}-1\right)\right)$ for all $j \geq 0$.

Bruner, using a computer, has verified that this conjecture holds for pairs $i$, $j$ such that $i \leq 4$ and $\left|T\left(i ; 2\left(2^{j+1}-1\right)\right)\right| \leq 255$.

The pattern of these formulas for $\chi T\left(j ; 2^{k}\right), k=0,1$, breaks down for $k=2$; it is not true that $\chi T(1 ; 4)=\langle 12\rangle$.

Theorem 3.1 and Conjecture 1.2, if it is true, allow us to identify new families of hit monomials, as we discuss in $\S 4$.

\section{TeChNicAl Lemma}

We begin by recalling a proposition from the proof of Theorem 1.1. For integers $a=\sum a_{i} 2^{i}$ and $b=\sum b_{i} 2^{i}$, where $a_{i}, b_{i} \in\{0,1\}$, we say $a$ dominates $b$ if $a_{i} \geq b_{i}$ for all $i$, and write $a \succeq b$.

Proposition 2.1 [D]. $\langle m\rangle \chi\langle n\rangle=\sum\left\{\langle R\rangle:|R|=m+n ;|R|+\sum r_{i} \succeq 2 m\right\}$.

An argument similar to Davis's describes the result when the order of factors is reversed.

Proposiiion 2.2. $\chi\langle n\rangle \cdot\langle m\rangle=\sum\left\{\langle R\rangle:|R|=m+n ; \sum r_{i} \succeq m\right\}$.

Note that in both cases, the question of whether an $\langle R\rangle$ of the relevant degree appears as a summand depends only on $\sum r_{i}$. The formulas are very similar, and the following lemma, used repeatedly in the inductive proof of Theorem 3.1 , combines them to give a way of pushing a factor involving $\chi$ through a product. Specifically, it permits us, in certain cases, to write $\langle m\rangle \chi\langle n\rangle$ as the sum of $\chi\langle p\rangle \cdot\langle q\rangle$ and two terms of the form $\left\langle m^{\prime}\right\rangle \chi\left\langle n^{\prime}\right\rangle$, which with luck will be more tractable than the original $\langle m\rangle \chi\langle n\rangle$.

Lemma 2.3. Suppose that $k, l, m$, and $n$ are nonnegative integers such that:

(1) $k>l$,

(2) $m+n=2^{k}-2^{l}$,

(3) $m<2^{k-1}$,

(4) $m \equiv 0\left(\bmod 2^{l}\right)$. 
Then

$$
\begin{aligned}
S q(m) \cdot \chi S q(n)= & \chi S q\left(n-m-2^{l}\right) \cdot S q\left(2 m+2^{l}\right)+S q\left(m+2^{k-1}+2^{l-1}\right) \\
& \cdot \chi S q\left(n-2^{k-1}-2^{l-1}\right)+S q\left(\frac{m+n}{2}\right) \cdot \chi S q\left(\frac{m+n}{2}\right),
\end{aligned}
$$

with the understanding that $\chi S q(s)=0$ if $s<0$, and that the rightmost two terms vanish when $l=0$.

Proof. For simplicity, we will refer to the $i$ th term in the equation above as $Q_{i}$. To prove the lemma, it is enough to show that $\mathscr{B}\left(Q_{1}\right)=\mathscr{B}\left(Q_{2}\right) \amalg \mathscr{B}\left(Q_{3}\right)$ $\amalg \mathscr{B}\left(Q_{4}\right)$, where $X \amalg Y$ is the symmetric difference of the two sets. For $S q(T)=S q\left(t_{1}, t_{2}, \ldots\right)$ a basis element of degree $|S q(T)|=2^{k} K-2^{l}$, write $L_{T}=2^{k} K-2^{l}+\sum t_{i}$ and $R_{T}=\sum t_{i}$. By Propositions 2.1 and 2.2, $S q(T) \in$ $\mathscr{B}\left(Q_{1}\right)$ (resp. $\mathscr{B}\left(Q_{2}\right)$, resp. $\mathscr{B}\left(Q_{3}\right)$, resp. $\left.\mathscr{B}\left(Q_{4}\right)\right) \Leftrightarrow L_{T} \succeq 2 m$ (resp. $R_{T} \succeq$ $2 m+2^{l}$, resp. $L_{T} \succeq 2^{k}+2 m+2^{l}$, resp. $\left.L_{t} \succeq 2^{k}-2^{l}\right)$. By (3) and (4) above, $\mathscr{B}\left(Q_{3}\right)$ and $\mathscr{B}\left(Q_{4}\right)$ are $\subseteq \mathscr{B}\left(Q_{1}\right)$. Writing $L_{T}=\left(R_{T}-2^{l}\right)+2^{k} K$ and using (3) and (4), one finds that also $\mathscr{B}\left(Q_{2}\right) \subseteq \mathscr{B}\left(Q_{1}\right)$. Similarly, expressing $R_{T}$ in terms of $L_{T}$, one may check that $\mathscr{B}\left(Q_{1}\right) \subseteq \mathscr{B}\left(Q_{2}\right) \amalg \mathscr{B}\left(Q_{3}\right) \amalg \mathscr{B}\left(Q_{4}\right)$. Observe that if $S q(T) \in \mathscr{B}\left(Q_{1}\right)$, then $S q(T) \in \mathscr{B}\left(Q_{4}\right) \Leftrightarrow \sum t_{i}<2^{l}$. Finally, note that $\mathscr{B}\left(Q_{1}\right)=B\left(Q_{2}\right)$ when $l=0$. This proves the lemma.

\section{Proof of THE THEOREM}

We are now ready to prove the main result of this paper.

Theorem 3.1. For all integers $i$ and $j$, we have $\chi T\left(j ; 2^{i+1}-1\right)=T\left(i ; 2^{j+1}-1\right)$. Proof. Clearly it suffices to prove the theorem under the assumption that $i \leq j$. Theorem 2.1 gives the result for $i=0$ and all $j$, so we proceed inductively by assuming that $\chi T\left(\hat{\imath} ; 2^{\hat{\jmath}+1}-1\right)=T\left(\hat{\jmath} ; 2^{\hat{\imath}+1}-1\right)$ for $\hat{\imath} \leq i-1$ and all $\hat{\jmath}$ and for $\hat{\imath}=i$ and all $\hat{\jmath} \leq j-1$. The inductive proof will draw on the following remark: under the above assumptions,

$$
\begin{array}{ll}
\chi S q\left(2^{l-1} K\right) \cdot T\left(l-1 ; 2^{i+1}-1\right)=0 & \text { for all } K \equiv 1(\bmod 2) \\
& \text { and all } 1 \leq l \leq i
\end{array}
$$

For we have

$$
\begin{aligned}
\chi S q & \left(2^{l-1} K\right) \cdot T\left(l-1 ; 2^{i+1}-1\right)=\chi\left[\chi T\left(l-1 ; 2^{i+1}-1\right) \cdot S q\left(2^{l-1} K\right)\right] \\
& =\chi\left[T\left(i ; 2^{l}-1\right) \cdot S q\left(2^{l-1} K\right)\right] \\
& =\chi\left[T\left(i-1 ; 2\left(2^{l}-1\right)\right) S q\left(2^{l}-1\right) \cdot S q\left(2^{l-1} K\right)\right]
\end{aligned}
$$

and it follows from the Adem relations that $S q\left(2^{l}-1\right) S q\left(2^{l-1} K\right)=0$. This verifies (1).

Now consider

$$
\begin{aligned}
\chi T\left(i ; 2^{j+1}-1\right) & =\chi\left[T\left(i-1 ; 2^{j+1}-1\right)\right] \cdot \chi\left\langle 2^{i}\left(2^{j+1}-1\right)\right\rangle \\
& \stackrel{\text { ind }}{=} T\left(j ; 2^{i}-1\right) \cdot \chi\left\langle 2^{i}\left(2^{j+1}-1\right)\right\rangle .
\end{aligned}
$$

Beginning from the right, we multiply $\chi\left\langle 2^{i}\left(2^{j+1}-1\right)\right\rangle$ by the successive terms $\left\langle 2^{l}\left(2^{i}-1\right)\right\rangle, 0 \leq l \leq j$, of $T\left(j ; 2^{i}-1\right)$. At the cost of an error term, Lemma 2.3 allows us to push the factor involving $\chi$ past the $l$ th singleton, transforming 
both in the process. Furthermore, (1) ensures that the error term vanishes upon multiplication by the $(l-1)$-singletons to its right. Namely, we prove that

$$
\begin{aligned}
& T\left(l ; 2^{i}-1\right) \cdot \chi\left\langle 2^{i}\left(2^{j+1}-1\right)\right\rangle \\
& \quad=\chi\left\langle 2^{i}\left(2^{j+1}-2^{l+1}\right)\right\rangle \cdot T\left(l ; 2^{i+1}-1\right) \quad \text { for } 0 \leq l \leq j .
\end{aligned}
$$

The case $l=0$ follows from Lemma 2.3. Suppose then that the claim holds for $l-1$. Then, as $T\left(l ; 2^{i}-1\right)=\left\langle 2^{l}\left(2^{i}-1\right)\right\rangle \cdot T\left(l-1 ; 2^{i}-1\right)$, we have

$$
\begin{aligned}
T(l & \left.; 2^{i}-1\right) \cdot \chi S q\left(2^{i}\left(2^{j+1}-1\right)\right) \\
= & S q\left(2^{l}\left(2^{i}-1\right)\right) \cdot \chi S q\left(2^{i}\left(2^{j+1}-2^{l}\right)\right) \cdot T\left(l-1 ; 2^{i+1}-1\right) \quad \text { [induction] } \\
= & {\left[\chi S q\left(2^{i}\left(2^{j+1}-2^{l+1}\right)\right) \cdot S q\left(2^{l}\left(2^{i+1}-1\right)\right)\right] \cdot T\left(l-1 ; 2^{i+1}-1\right) \quad \text { [Lemma 2.3] } } \\
& +\left[S q\left(2^{i+j}+2^{i+l}-2^{l-1}\right) \cdot \chi S q\left(2^{i+j}-2^{i+l}-2^{l-1}\right)\right] \cdot T\left(l-1 ; 2^{i+1}-1\right) \\
& +\left[S q\left(2^{i+j}-2^{l-1}\right) \cdot \chi S q\left(2^{i+j}-2^{l-1}\right)\right] \cdot T\left(l-1 ; 2^{i+1}-1\right) .
\end{aligned}
$$

But the second and third summands vanish by (1), so we have

$$
\begin{aligned}
T(l ; & \left.2^{i}-1\right) \cdot \chi S q\left(2^{i}\left(2^{j+1}-1\right)\right) \\
& =\chi S q\left(2^{i}\left(2^{j+1}-2^{l-1}\right)\right) \cdot\left[S q\left(2^{l}\left(2^{i+1}-1\right)\right) T\left(l-1 ; 2^{i+1}-1\right)\right] \\
& =\chi S q\left(2^{i}\left(2^{j+1}-2^{l-1}\right)\right) \cdot T\left(l ; 2^{i+1}-1\right) .
\end{aligned}
$$

This establishes (3). Finally, taking $l=j$, we find that

$$
\begin{aligned}
\chi T\left(i ; 2^{j+1}-1\right) & =T\left(j ; 2^{i}-1\right) \cdot \chi\left\langle 2^{i}\left(2^{j+1}-1\right)\right\rangle \\
& =\chi\langle 0\rangle \cdot T\left(j ; 2^{i+1}-1\right) .
\end{aligned}
$$

This proves the theorem.

\section{Hit elements}

In [K] Kraines gives a proof that the excess ex $(\chi\langle n\rangle)$ is given by $\mu(n)$, where $\mu(n)$ is the number of summands in the most efficient way of writing $n$ as a sum of numbers of the form $2^{i}-1$; that is, $\mu(n)=\min \left\{m: n=\sum_{i=1}^{n}\left(2^{k_{i}}-1\right)\right.$ for some integers $k_{i}$ \}. The following generalization follows immediately from Theorem 3.1:

Corollary 4.1. $\operatorname{ex}\left(\chi T\left(i ; 2^{j+1}-1\right)\right)=2^{i+1}-1 \quad\left(=\left(2^{i+1}-1\right) \mu\left(2^{j+1}-1\right)\right)$.

In view of its consequences in the study of hit monomials, described below, Corollary 4.1 leads to

Conjecture 4.2. $\operatorname{ex}(\chi T(i ; a))=\left(2^{i+1}-1\right) \mu(a)$.

The case $a=2, i$ arbitrary, would follow from Conjecture 1.2. Using a computer, Bruner has verified Conjecture 4.2 for all pairs $(i, a)$ such that $a \leq 31$ and $|T(i ; a)| \leq 255$.

The connection of Conjecture 4.2 with hit monomials is as follows: Recall that $\alpha(n)$ denotes the number of 1's in the binary expansion of the integer $n$. In [W] Wood extends and proves a conjecture due to Peterson. 
Theorem 4.3 [W]. Let $M$ be a monomial of degree $|M|=d$, and suppose that $e$ of its exponents are odd. If $\alpha(d+e)>e$, then $M$ is hit.

The proof involves writing $M$ in the form $E F^{2}=E \cdot\langle f\rangle(F)$, where $E$ is squarefree of degree $e$ and $f=|F|$, and showing that modulo hit elements, $E \cdot \theta(F) \equiv \chi \theta(E) \cdot F$ for any $\theta \in \mathscr{A}(2)$. The result then follows from the fact that $\operatorname{ex}(\chi\langle f\rangle)=\mu(f)$; one must check that the assumption $\alpha(d+e)>e$ implies that $\mu(f)>e$.

Singer has conjectured a generalization of Theorem 4.3, using a condition which involves not the degree of the squarefree part of $M$ but rather the degree of the $2^{k+1}$-powerfree part. That is, write $M=E F^{2^{k+1}}$ where $E$ contains no $2^{k+1}$-powers, and let $e=|E|, f=|F|$. The conjecture can be paraphrased in part as follows.

Conjecture 4.4 [Si]. Suppose that $M$ is a monomial with decomposition $M=$ $E F^{2^{k+1}}$ as above. If $e<\left(2^{k+1}-1\right) \mu(f)$, then $M$ is hit.

This time, we have that

$$
E F^{2^{k+1}}=E \cdot\left[\left\langle 2^{k} f\right\rangle \cdots\langle 2 f\rangle\langle f\rangle(F)\right]=E \cdot[T(k ; f)(F)],
$$

which modulo hit elements is congruent to $[\chi T(k ; f)(E)] \cdot F$; but this element vanishes if $\operatorname{ex}(\chi T(k ; f))>|E|$, so we see that Conjecture 4.2 implies Conjecture 4.4. In the cases for which Conjecture 4.2 has been verified, we can state the following.

Theorem 4.5. Suppose that $M$ is a monomial with decomposition $E F^{2^{k+1}}$ as above and that $e<\left(2^{k+1}-1\right) \mu(f)$. If $f=2$ or $f=2^{i}-1$ for some $i$, or if $(k, f)$ satisfies $f \leq 31$ and $\left(2^{k+1}-1\right) f<255$, then $M$ is hit.

In unpublished work, Singer has verified Conjecture 4.4 for $k=1$ and all $f$ using different techniques.

\section{REFERENCES}

[D] Donald M. Davis, The antiautomorphism of the Steenrod algebra, Proc. Amer. Math. Soc. 44 (1974), 235-236.

[K] D. Kraines, On excess in the Milnor basis, Bull. London Math. Soc. 3 (1971), 363-365.

[M] John Milnor, The Steenrod algebra and its dual, Ann. of Math. (2) 67 (1958), 150-171.

[P] F. P. Peterson, A-generators for certain polynomial algebras, Math. Proc. Cambridge Philos. Soc. 105 (1989), 311-312.

[SE] N. E. Steenrod and D. B. A. Epstein, Cohomology operations, Princeton Univ. Press, Princeton, NJ, 1962.

[S] W. Singer, On the action of Steenrod squares on polynomial algebras, Proc. Amer. Math. Soc. 111 (1991), 577-583.

[W] R. M. W. Wood, Steenrod squares of polynomials and the Petersen conjecture, Math. Proc. Cambridge Philos. Soc. 105 (1989), 307-309.

Department of Mathematics, University of Michigan, Ann Arbor, Michigan 48109

E-mail address: Judith.Silverman@math.lsa.umich.edu 